\title{
Multi-focus Image Fusion using an Improved Differential Evolution Algorithm and Adaptive Block Mechanism
}

\author{
Feng Yong ${ }^{1,2, *}$, Li Tiezhu ${ }^{1,2}$ and Zhou Shangbo ${ }^{1,2}$ \\ ${ }^{1}$ Key Laboratory of Dependable Service Computing in Cyber Physical Society, Ministry of Education, Chongqing University, \\ Chongqing 400030, China \\ ${ }^{2}$ College of Computer Science, Chongqing University, Chongqing 400030, China
}

Received: 7 Sep. 2013, Revised: 5 Dec. 2013, Accepted: 6 Dec. 2013

Published online: 1 Sep. 2014

\begin{abstract}
Multi-focus image fusion based on differential evolution algorithm has been demonstrated to be a simple and efficient method. However, several some problems remain. First, the fusion rulesin which the best block size is selected is only based on the original differential evolution algorithm, which easily falls into the local convergence and thus affects the global search ability. When the sharpness values of the corresponding blocks are equal, the method becomes ineffective, because the block effect is enhanced. Secondthe algorithm ignores image size. Thus, calculating larger pictures becomes complex and time consuming. Clear and fuzzy areas are further divided, thus causing unnecessary calculations. Therefore, a multi-focus images fusion method based on an improved differential evolution algorithm and adaptive block mechanism is presented. First, the source images are divided by a fixed size once. Then, the boundary region is searched to find adaptive blocks using the improved differential evolution algorithm. If the sharpness values of the corresponding blocks are equal, the extends block mechanism is applied to determine the block with the highest sharpness value. Experimental results show that the improved algorithm can obtain better fusion effects and consume less time compared with the original differential evolution algorithm.
\end{abstract}

Keywords: Multi-focus image fusion,differential evolution algorithm,extends block mechanism,adaptive block mechanism.

\section{Introduction}

With the development of image sensors, the application of image technology has become increasingly widespread $[1,2,3]$. This thesis focuses on multi-focus image fusion using image block segment [5] and takes advantage of the characteristics of multi-focus images. The main objective of this work is to divide the source images into blocks, then select the corresponding blocks with the highest sharpness values to construct the resultant fused image. The method is computationally simple and has a good fusion effect.

The differential evolution (DE) [6] algorithm is employed to determine the suitable sizes of blocks in multifocus image fusion. However, several some problems remain. First, the fusion rule in which the method selects the best block size is only based on the original DE algorithm, which easily falls into the local convergence. As the DE evolution algorithm randomly initializes the population, its global search ability may be seriously affected. When the sharpness values are equal among the corresponding blocks, the method used to determine the sharpest image block becomes ineffective, because the values of the source images may change and the block effect may be enhanced. Secondly, the algorithm ignores image size and deals with all pictures in the same manner. Calculating large pictures thus becomes complex and time consuming. Images method also does not take full advantage of the characteristics of multi-focus images that are divided into clear, fuzzy and are further divided boundary regions. All clear and fuzzy areas are further divided, thus causing unnecessary calculations.

As for the fusion rules to improve the performance of the DE algorithm, several approaches have been proposed; examples include a combination method of DE and an estimation of distribution algorithm to improve global search ability in [7], a hybrid method of DE and ant colony algorithm to accelerate convergence speed in [8], and initialization using the second difference method to accelerate convergence speed in [9]. However, these methods have several issues, such as complicated programming, and compu-

\footnotetext{
*Corresponding author e-mail: fengyong@ @ cqu.edu.cn
} 
tational complexity. In the present study, an enhanced DE algorithm is proposed to improve global and local search abilities, via a computationally simple approach. Spatial Frequency, Variance and Sum-modified Laplacian [4] are used as criterion functions to measure the sharpness of image blocks. If the sharpness values are of blocks $A_{i}$ and $B_{i}$ are equal, then the $i t h$ block of the fused image as: $F_{i}=\left(A_{i}+B_{i}\right) / 2$. However, the values of the corresponding pixels in $A_{i}$ and $B_{i}$ may not be completely equal. Thus, some original pixel values are changed in the fused image and the block effect is enhanced. One randomly selected block is also inappropriate [10]. The present work presents a simple and effective approach to solve these problems, that is, the extends block selection mechanism.

Although multi-focus image fusion based on the adaptive block algorithm [11,12] only splits the image in the border region, the processing of the image block is short, and the computational complexity and time cost are significantly reduced. Clear and fuzzy regions are divided by progressive approximation, which is based on the mathematical concept of differential. Therefore, a multi-focus image fusion technique based on an improved DE algorithm(IDEA) and adaptive block mechanism(ADBM) is presented. First, the source images are divided by a fixed size once. Then, the boundary region is searched to find adaptive blocks using IDEA. If the sharpness values of the corresponding blocks are equal, then extends block mechanism is applied to determine the block with the highest sharpness value. Experimental results show that this algorithm can obtain better fusion effects, be calculated more efficiently, and consume less time compared with the original DE algorithm.

The main contribution of this work is its presentation of an IDEA and extends block mechanism, for multi-focus images fusion using ADBM. The rest of the paper is organized as follows. Section 2 introduces the IDEA. Section 3 presents the proposed extends block mechanism. Section 4 describes the adaptive block algorithm-based multifocus images fusion method. Second 5 introduces the use of IDEA and adaptive block mechanism in multi-focus image fusion. Section 6 provides the experimental results and discussion, and Section 7 concludes the study.

\section{Improved differential evolution algorithm}

The IDEA is proposed in this section, relies on the characteristics that are considered in determining the optimal block size( $m$ and $n)$. Such characteristics include a solution comprising two integer values and a small population. The principle of the IDEA is the same as that of DE, but IDEA initializes the population at different intervals and introduces population determination.

1) Initial population at different intervals. First, the ranges of $\mathrm{m}$ and $\mathrm{n}$ are divided into $N P$ (population size) intervals and the average of the individual $(m, n)$, respectively. Then, an individual in each corresponding interval of $\mathrm{m}$ and $\mathrm{n}$ to form the initial population.
2) Population determination. When all individuals are equal in the population, we reserve an individual $X_{i}$ and randomly initialize the NP-1 individuals, which makes up the next generation together with $X_{i}$ to improve the convergence speed, let the re-initialization of the N-1 individuals and $X_{i}$ be within the same interval.

\subsection{IDEA framework}

(1) Population size $(N P)$, scaling factor $(F)$, and crossover probability $(C R)$ as well as termination criteria are determined.

(2) Initial population at different intervals. If preliminary knowledge about the problem to be optimized is absent, then the first population of solutions can be generated at different intervals as follows:

$X_{i, G}=X_{i(L)}+\operatorname{rand}_{i}[0,1]\left(X_{i(H)}-X_{i(L)}\right)$,

where $X_{i(L)}$ and $X_{i(H)}$ are the lower and higher boundaries, respectively, at different intervals of two-dimensional vector $X_{i}=\left\{m_{i}, n_{i}\right\}$.

(3) Mutation. For each target vector , $i=1,2, \ldots, \mathrm{NP}$, a mutant vector is generated according to

$V_{i, G+1}=X_{r 1, G}+F\left(X_{r 2, G}-X_{r 3, G}\right)$,

where $\mathrm{i}, \mathrm{r} 1, \mathrm{r} 2$ and $\mathrm{r} 3$ are reciprocally different random integer indices selected from $\{1,2, \ldots, N P\}$. Therefore, the population size $N P$ must be greater than $3 . F \in[0,2]$ is a real constant positive weighting factor that controls the amplification of the added differential variation of $\left(X_{r 2(G)}-\right.$ $\left.X_{r 3(G)}\right)$.

(4) Crossover. To increase the diversity of the population, the trial vector is formed by:

$$
U_{j, i, G+1}= \begin{cases}V_{j, i, G+1} & \text { if } \operatorname{rand}_{j, i} \leq \mathrm{CR} \text { or } j=I_{\text {rand }} \\ X_{j, i, G} & \text { if } \operatorname{rand}_{j, i}>\mathrm{CR} \text { or } j \neq I_{\text {rand }},(3)\end{cases}
$$

where $j=1,2, \ldots, D$ (D is the number of vectors, which is $2(\mathrm{~m}, \mathrm{n})$ in this paper); $\operatorname{rand}_{j, i} \in(0,1)$ is a random real number, $C R \in[0,1]$ is a predefined crossover constant, and $I_{\text {rand }} \in[1,2, \ldots, D]$ is a randomly chosen index. $I_{\text {rand }}$ ensures that $V_{i, G+1} \neq X_{i, G}$.

(5) Selection. The approach must decide which of the two vectors, i.e., $U_{i, G+1}$ or $X_{i, G}$, should be a member of new generation $G+1$. The vector with a fitter than its counterpart value is chosen.

(6) Population determination. If all individuals are equal in the population, we reserve an individual $X_{i}$ and randomly re-initialize the NP-1 individuals (the $N P-1$ and $X_{i}$ within the same interval.).

(7) Termination test. Step (3) is performed until a predefined condition is satisfied.

\subsection{Comparison with the original DE algorithm}

Figures 1 and 2 show the major differences between the original DE algorithm and the IDEA. That is, the IDEA 
Step 1: Randomly initialize population, and set $\mathrm{g}=1$

Step 2: If $\mathrm{g}=\mathrm{MaxGens}$, output $\mathrm{Xi}$, else go on

Step 3: Mutation

Step 4: Crossover

Step 5: Selection

Step 6: $\mathrm{g}++$, go on step 2

Figure 1 The flow chart of the standard DE algorithm.

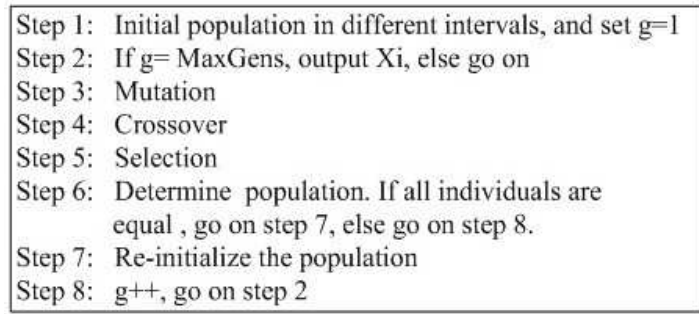

Figure 2 The flow chart of the IDEA.

initializes the population at different intervals and introduces population.

1) The original DE algorithm randomly initializes the population. Individuals in the population may sometimes differ, but the differential variation is very small. When they mutate, these individuals undergo little change. In this case, falling into the local convergence is easy with the constant iterations. This condition affects the global search ability of the algorithm. By contrast, the IDEA initializes the population at different intervals ,thereby ensuring a large number of differences. Therefore, the differential variation in individuals is large at the early iterations. The mutation results in a considerable change in the individuals, This condition allows the IDEA to perform a wide search with a reasonable number of iterations, thus improving its global search capability.

2) After the random initialization of the population using the original DE algorithm, the differences among the individuals including, the differential variation in individuals, decrease with the constant iterations. Therefore, the individual differences also decrease and reach local convergence upon mutation. The algorithm performs the search only once at the local range. By contrast, IDEA determines the population. When the IDEA converges into a solution and reserves it, then one or more local search thereby improving in the range of the solution, thereby improving the local search ability of the algorithm.

\section{Extends block mechanism}

\subsection{Criterion functions}

This section explains the criterion functions, which are employed to measure the sharpness of images.

(1) SF. The SF indicates the overall activity level in an image. The criterion is computed as

$S F=\sqrt{C^{2}+R^{2}}$,

where $C$ and $R$ are the row and column gradients, respectively, and defined as

$C=\left[\frac{1}{M \times N} \sum_{i=1} \sum_{j=1}[f(i, j)-f(i-1, j)]^{2}\right]^{1 / 2}$,
$R=\left[\frac{1}{M \times N} \sum_{i=1} \sum_{j=1}[f(i, j)-f(i, j-1)]^{2}\right]^{1 / 2}$,

Let $f(i, j)$ be the gray level intensity of the pixel $(i, j)$, and let $M$ and $N$ be the dimensions of the images.

(2) VAR. The gray level image variance can be computed by subtracting the mean gray level intensity value from the gray level of each pixel. The focus measure in this case computed as:

$$
\begin{aligned}
& V A R=\frac{1}{M \times N} \sum_{i=1} \sum_{j=1}(f(i, j)-\bar{f})^{2}, \\
& \mathbf{f}=\frac{1}{M \times N} \sum_{i=1} \sum_{j=1} f(i, j),
\end{aligned}
$$

where $\bar{f}$ is the average gray level over the image region.

(3) SML. Sum-modified Laplacian differs from the usual Laplacian terms of the summation of the partial second derivatives; in the former, the absolute values of the partial second derivatives are summed instead of their actual values.

$$
\begin{aligned}
& \nabla^{2} f(i, j)=\left|\frac{\partial^{2} f(i, j)}{\partial i^{2}}\right|+\left|\frac{\partial^{2} f(i, j)}{\partial j^{2}}\right| \\
& =|2 f(i, j)-f(i-1, j)-f(i+1, j)| \\
& +|2 f(i, j)-f(i, j+1)-f(i, j-1)| .
\end{aligned}
$$

The focus measure is given by:

$S M L=\sum \sum \nabla^{2} f(i, j)$

The three functions above can all be used to measure the sharpness of an image, a large value indicates a clear image. 


\subsection{Extends Block Mechanism}

1) Extends block method

The use $S F, V A R$ and $S M L$ are used to measure the sharpness of image blocks. If the sharpness values of the corresponding blocks $A_{i}$ and $B_{i}$ are equal, then the same value is extended to adjacent block $C$, which is also the sharper image block. The sharpness values of the two larger blocks $A_{i} C$ and $B_{i} C$ are compared to determine the sharper image block $F_{i} C$.

2) Extends block theory

For example, the $S F$ is used to measure the sharpness of image blocks. Assume the following image blocks: $A_{i}=[12,14,16,18]$ and $B_{i}=[34,36,38,40]$. Then, $S F\left(A_{i}\right)=S F\left(B_{i}\right)=1.7321$. Clearly, the sharpness values of blocks $A_{i}$ and $B_{i}$. Thus, the same size is extended to adjacent block $C=[28,30,25,41]$, which is also the sharper image block. Then, the sharpness values of the two larger blocks $A_{i} C$ and $B_{i} C$ are $S F\left(A_{i} C\right)=$ 13.3182 and $S F\left(B_{C}\right)=8.2082$, respectively. With the sharpness of the whole fused image determined, $A_{i}$ is selected as the sharper image block. If the corresponding pixels of $A_{i}$ and $B_{i}$ (with the same sharpness values) are not completely equal even after extending the same block $C$, then the criterion functions indicate that the sharpness values of blocks $A_{i} C$ and $B_{i} C$ must be different. However, the original method constructs $i t h$ block $F_{i} C$ of the fused image as: $F_{i}=\left(A_{i}+B_{i}\right) / 2, F_{i}=[23,25,27,29]$, $S F\left(C F_{i}\right)=7.8661$, but $S F\left(C F_{i}\right)<S F\left(A_{i} C\right)$. Despite $S F\left(C F_{i}\right)>S F\left(A_{i} C\right)$ on certain occasions, the values of the source images are still changed and increase the block effect.

\subsection{Characteristics of the extends mechanism}

(1) The approach is simple, easy to understand, and requires little computation.

(2) If the corresponding pixels of $A_{i}$ and $B_{i}$ (with the same sharpness values) are not completely equal even after extending the same block $C$, the values of blocks $A_{i} C$ and $B_{i} C$ must be different.

(3) The extend block mechanism considers the sharpness of the whole fused image.

(4) The extend block mechanism does not change the values of the original images. This characteristic reduces the block effect and enhances the fused image.

\section{Multi-focus image fusion using adaptive block mechanism}

This section explains the use of ADBM in multi-focus images fusion. In most cases, the differences in the fusion results with input images and the sensitivity of the human visual system to the edges of the image region cannot be ignored (Fig. 4.1). Generally, an image is divided into

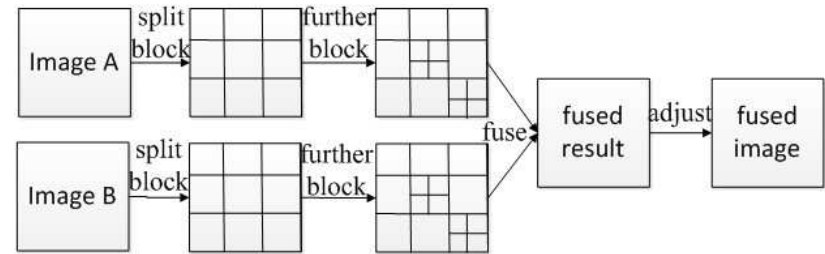

Figure 3 The ADBM fusion method flowchart.

three areas: sharp, blurred, and boundary regions. The optimal block size is different for different regions of the image. For the sharp and blurred regions, the division of the image must be large because the corresponding characteristics of the sub-block are significantly different. For the boundary region, a small size is necessary. Thus, the sharp and blurred regions are located at different sub-blocks, and this condition can improve the quality of the fused image. The ADBM fusion flowchart is shown in Figure 3.

The basic steps of the ADBM are described as follows:

(1) Divide input images A and B into non-overlapping blocks with a size of $m \times n$. The first initialized block size is determined by the input images; the size must not be too small or too large. Take for example an input image measuring $256 \times 256$. In this case, the decomposition sizes can generally be $32 \times 32,32 \times 16,16 \times 32$ and $16 \times 16$. Refer to the ith image blocks of images $\mathrm{A}$ and $\mathrm{B}$ denoted as $A_{i}$ and $B_{i}$, respectively.

(2) Apply the criterion function on $A_{i}$ and $B_{i}$ to calculate the sharpness values of the blocks, and denote the results of $A_{i}$ and $B_{i}$ as $f\left(A_{i}\right)$ and $f\left(B_{i}\right)$, respectively.

(3) Compare the sharpness values of two corresponding blocks $A_{i}$ and $B_{i}$ to determine the sharper image block, and construct $i$ th block $F_{i}$ of the fused image as:

$$
F_{i}=\left\{\begin{array}{lll}
A_{i} & & f\left(A_{i}\right)>f\left(B_{i}\right)+T H \\
B_{i} & & f\left(A_{i}\right)<f\left(B_{i}\right)+T H \\
\text { further split } & \text { otherwise }
\end{array}\right.
$$

where TH is the customized threshold.

(4) After applying (3-1) for each corresponding block of the source images, further divide the image blocks by repeating steps 2 and 3 until a predefined condition is satisfied.The minimum block as the termination criteria is set to $4 \times 4$ in the present work.

(5) Check the consistency of the fusion results, i.e. ,if an image block in the fusion results from image $\mathrm{A}$, and if the adjacent image blocks result from image $\mathrm{B}$, then change the image block to the corresponding block of image $\mathrm{B}$, The $3 \times 3$ neighborhood voting method is used in this work. 


\section{Multi-focus image fusion using improved differential evolution algorithm and adaptive block mechanism}

A multi-focus images fusion using IDEA and ADBM is proposed in this section. The method relies on the characteristics of multi-focus images. The basic steps of the proposed method are as follows:

(1) Divide input images A and B into non-overlapping blocks with a size of. The first initialized block size is determined by the input images, the size must not be too small, or too large. Take for example an input image measuring $256 \times 256$. In this case, the decomposition size can generally be $64 \times 64$. Refer to the ith image blocks of images $\mathrm{A}$ and $\mathrm{B}$ denoted as $A_{i}$ and $B_{i}$, respectively.

(2) Reference the same steps(2) from Section 4.

(3) Compare the sharpness values of two corresponding blocks $A_{i}$ and $B_{i}$ to determine the sharper image block, and construct $i$ th block $F_{i}$ of the fused image as

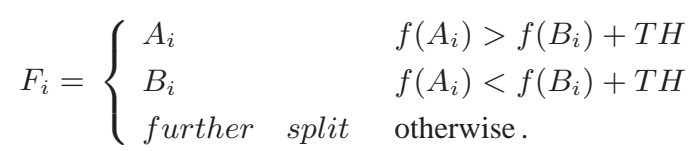

where $\mathrm{TH}$ is the customized threshold. TH selection is very critical because the selected TH can which directly affect the quality of the image fusion effects. However, $\mathrm{TH}$ selection is very difficult because of the distribution of sharp, blurred, and boundary regions and the differing proportions of different multi-focus images. TH selection in this work is performed via an experimental method.

Calculate the corresponding sub-block of the SF difference array $D T_{i}$. Then, sort $D T_{i}$ in ascending order to obtain a new array of $D T$. To calculate $A-B$, select the maximum value of the above difference.

(4) If the sharpness values of all the corresponding subblocks in the segmentation of source image segmentation slightly differ, the above blocks must be divided further, and all sub-blocks are assumed to be at the junction. Then, proceed to step 1 and re-divide the image blocks; the size should be half of the original. Otherwise, go to step 4.

(5) Continuously divide the image blocks using the IDEA and extends block mechanism, as described in the second and third part of this article.

In the proposed method, the source images divided once using the fixed size.The boundary region is then searched using the IDEA to find the adaptive blocks. If the sharpness values of the corresponding blocks are equal, then the extends block mechanism is applied to determine the block with the highest sharpness value. Experimental results show that the proposed algorithm can obtain better fusion effects, be calculated more efficiently, and consume less time compared with the original DE algorithm.

\section{Experimental results and discussion}

In this section, several experimental results are presented to demonstrate the performance of the IDEA and the ex-

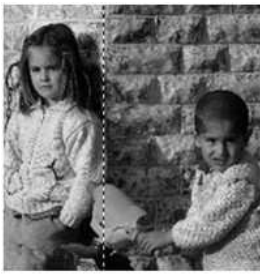

(a)

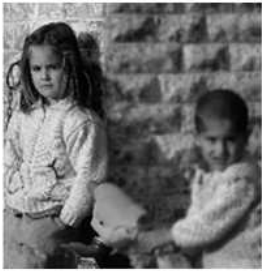

(b)

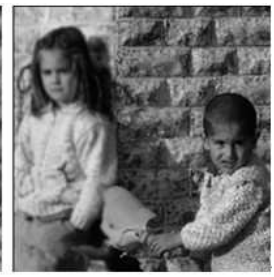

(c)
Figure 4 Kids images: (a) reference image; (b) focus on the girl; (c) focus on the boy.

tends block method in image fusion. Experiments were carried out on artificially produced multi-focus images. The following sharpness functions are measured: $S F, V A R$, $S M L$. Other parameters are as follows:population size NP $=5$, maximum number of generations $G_{\max }=100$, assessment measures mean square error (MSE) and peak signalto-noise ratio (PSNR).

(1) $M S E$ is a commonly used reference-based assessment metric that is defined as follows:

$M S E=\frac{1}{M \times N} \sum_{i=1}^{M} \sum_{j=1}^{N}(R(i, j)-F(i, j))^{2}$.

where $R(i, j)$ and $F(i, j)$ are the pixel values of the ideal reference and the obtained fused image, respectively. $M$ and $\mathrm{N}$ are the dimensions of the images. A small MSE value indicates a satisfactory fused image.

(2) $P S N R$ is a metric for the ratio of the maximum possible power of a signal to the power of corrupting noise that affects the fidelity of its representation. $P S N R$ is expressed as follows:

$$
P S N R=10 \log _{10}\left(\frac{1}{\frac{1}{M \times N} \sum_{i=1}^{M} \sum_{i=1}^{N}(R(i, j)-F(i, j))^{2}}\right)
$$

where $\mathrm{L}$ is the number of gray levels. A large PSNR value indicates satisfactory fusion results.

All the experiments are conducted on 8-bit gray images. The images are shown in Figures 4 to 6 . In (a), the images are all reference images (all in focus), (b) and (c) focus on different parts of the same image. The dashed line drawn on the reference image represents the border of the defocused regions. All the defocused versions are obtained by blurring the parts with a Gaussian of radius 1 .

(1) Test 1: Comparison of the former and the extends block mechanism fusion methods

(1) The quantitative comparison results of the original DE method and the extends block mechanism applied in Figures 4 to 6 are presented in Table 1. The comparison employs the MSE and PSNR metrics. (Note that both methods use the standard DE algorithm). The calculated MES values (1.52 for Figure 4 and 1.08 for Figure 6) of the extends block method are lower than those of the original DE method (2.88 for Figure 4 and 104.39 for Figure 6.) Meanwhile, the PSNR values (46.33 or Figure 4 and 


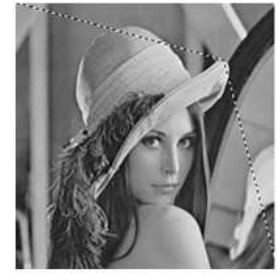

(a)

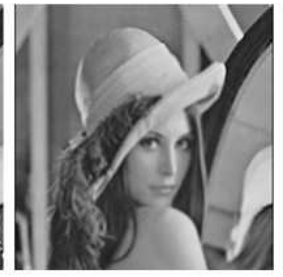

(b)

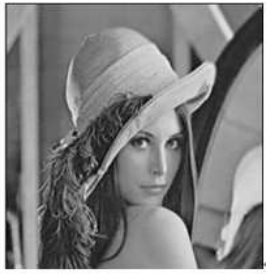

(c)
Figure 5 Lena images: (a) reference image; (b) focus on the right upper; (c) focus on the left bottom.

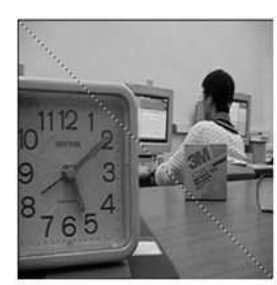

(a)

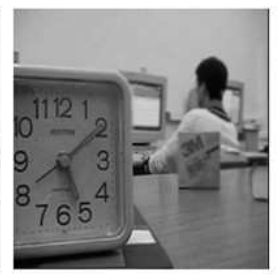

(b)

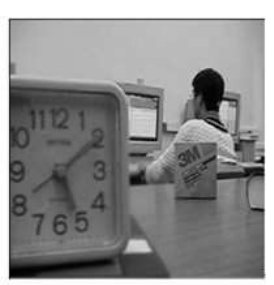

(c)
Figure 6 Clock images: (a) reference image; (b) focus on the clock; (c) focus on the man.

47.82 for Figure 6) of the extends block method are higher than those of the original DE method (43.56 for Figure 4 and 27.97 for Figure 6).

(2) The fusion results of the clock source images using the original DE and extends block methods are shown in Figures 7 (b) and (c). The differences between the fused images and the clock reference images (Figure 7 (a)) are also given in Figures 7 (d) and (e) for a visual comparison. As shown in Figure 7 (e), the extends block method obtains the fused image with the lowest reconstruction error.

Referring to the results presented in Table 1 and Figure 7 , we can conclude that the fusion results obtained by the extends block method is superior to the original DE method, particularly in cases in which the source images (e.g., Figure 6) have many corresponding segment image blocks with the same sharpness values. In addition to that, the values of MSE (1.56) and the PSNR (46.22) for Figure 5 are equal, because of the absence of corresponding blocks with the same sharpness values(VAR). This condition does not affect the correctness of the extends block method.

(2) Test 2: Comparison of the original DE and IDEA

(1) The fusion results of Figures 4 to 6 using the original DE algorithm and the IDEA are given in Table 2. The table also shows the mean of the MSE and PSNR results for 30 replicates. (Both methods use the extends block mechanism to select the blocks of the same sharpness). The calculated MES values (0.57, 0.50 and 1.12 for Figures 4, 5 and 6 , respectively) of IDEA are less than those
Table 1 Evaluation of the original DE and extends block method applied in the above images.

\begin{tabular}{|l|l|c|l|l|l|l|}
\hline Image & Functions & Method & F & CR & PSNR & MSE \\
\hline $\begin{array}{l}\text { Figure } \\
4\end{array}$ & SF & original DE & 0.3 & 0.6 & 43.56 & 2.88 \\
& & extends block & 0.3 & 0.6 & 46.33 & 1.52 \\
\hline $\begin{array}{l}\text { Figure } \\
5\end{array}$ & VAR & original DE & 0.6 & 0.3 & 46.22 & 1.56 \\
& & extends block & 0.6 & 0.3 & 46.22 & 1.56 \\
\hline $\begin{array}{l}\text { Figure } \\
6\end{array}$ & SML & original DE & 0.6 & 0.6 & 27.97 & 104.39 \\
& & extends block & 0.6 & 0.6 & 47.82 & 1.08 \\
\hline
\end{tabular}

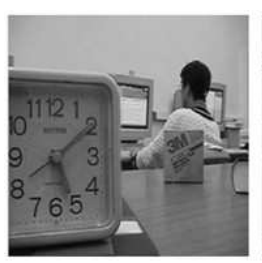

(a)

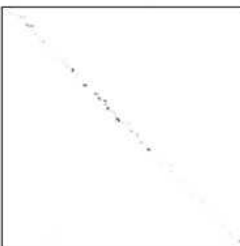

(d)

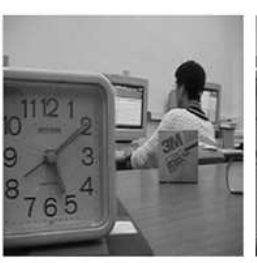

(b)

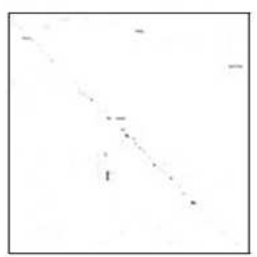

(e)

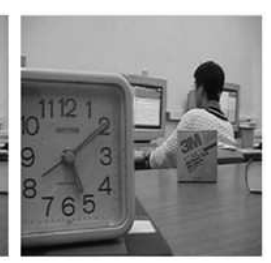

(c)
Figure 7 Clock images: (a)reference image; (b) fusion result of the original DE method; (c) fusion result of he extends block method; (d)differences between (a) and (c); (e)differences between (a) and (b).

of the original DE algorithm(1.08, 1.56, and 1.52 for Figures 4, 5 and 6 , respectively). Meanwhile, the values of the PSNR (47.64, 46.39 and 50.56 for Figures 4, 5 and 6, respectively) are higher than those of the original DE algorithm (46.33, 46.22 and 47.82 for Figures 4, 5 and 6, respectively).

(2) The comparison of the convergence results of the original DE algorithm and the IDEA applied in the fusion of the above images is illustrated in Figure 8. These figures represent the two images with the largest statistical numbers and sums. These values indicate the number of equal MSE values(Figures 4 to 6) in 32 experiments using both the original DE algorithm and the IDEA. The convergence of the IDEA is better that of the original DE algorithm. In particular, all the MES values of the IDEA are equal in the 32 experiments(Figure 9).

(3) Figure 9 shows the comparison of the global search 
Table 2 Evaluation of the original DE algorithm and IDEA applied in the images.

\begin{tabular}{|l|l|c|c|c|c|c|}
\hline Image & Functions & Methods & F & CR & PSNR & MSE \\
\hline $\begin{array}{l}\text { Figure } \\
4\end{array}$ & SF & Original DE & 0.3 & 0.6 & 46.33 & 1.52 \\
& & IDEA & 0.3 & 0.6 & 47.64 & 1.12 \\
\hline $\begin{array}{l}\text { Figure } \\
5\end{array}$ & VAR & Original DE & 0.6 & 0.3 & 46.22 & 1.56 \\
& & IDEA & 0.6 & 0.3 & 46.39 & 1.50 \\
\hline $\begin{array}{l}\text { Figure } \\
6\end{array}$ & SML & Original DE & 0.6 & 0.6 & 47.82 & 1.08 \\
& & IDEA & 0.6 & 0.6 & 50.56 & 0.57 \\
\hline
\end{tabular}
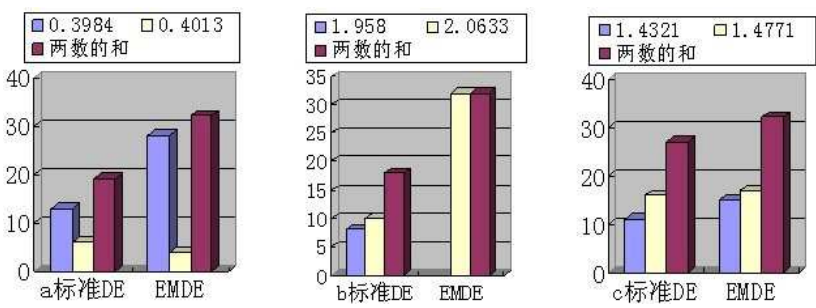

Figure 8 (a) Kids; (b) Lena; (c) Clock.

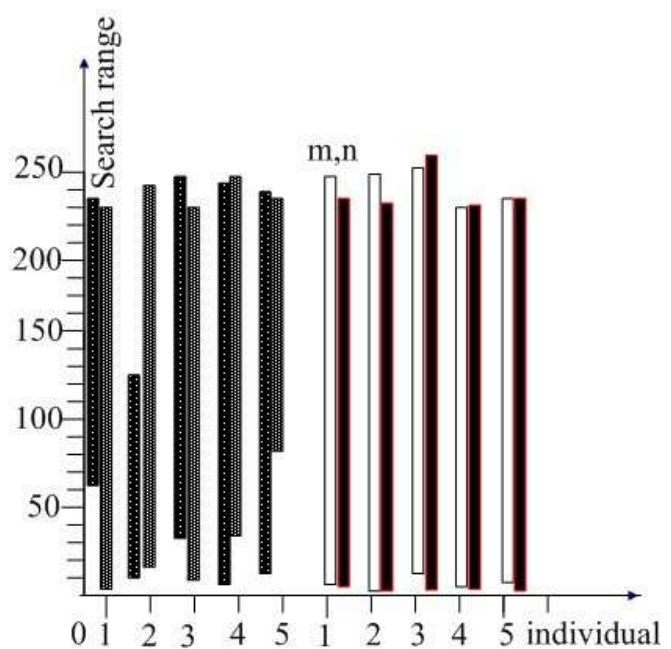

Figure 9 (left) Original DE algorithm;

(right) IDEA.

ability of the original DE algorithm and the IDEA applied in the fusion of Figure 4 (Kids image). This figure represents the $m$ and $n$ maximum search range (respectively) of the individual $(\mathrm{m}, \mathrm{n})$ in five random experiments on both the original DE algorithm and the IDEA. The global search range of the IDEA is obviously broader and more robust than that of the original DE algorithm.
Table 3 Comparison of the local search times of the original DE algorithm and the IDEA on the fusion of the Clock images (Figure 6)

\begin{tabular}{|c|cc|cc|}
\hline Label & \multicolumn{2}{|c|}{ IDEA (Optimal Size) } & Original DE(Optimal Size) \\
\hline 1 & 4 & $(9,7)$ & 1 & $(8,15)$ \\
\hline 2 & 3 & $(9,7)$ & 1 & $(9,7)$ \\
\hline 3 & 3 & $(9,7)$ & 1 & $(4,19)$ \\
\hline 4 & 3 & $(9,7)$ & 1 & $(8,10)$ \\
\hline 5 & 3 & $(9,7)$ & 1 & $(9,7)$ \\
\hline 6 & 3 & $(9,7)$ & 1 & $(4,29)$ \\
\hline 7 & 4 & $(9,7)$ & 1 & $(6,11)$ \\
\hline 8 & 3 & $(9,7)$ & 1 & $(8,10)$ \\
\hline 9 & 4 & $(9,7)$ & 1 & $(8,10)$ \\
\hline 10 & 3 & $(9,7)$ & 1 & $(8,6)$ \\
\hline
\end{tabular}

Table 4 Averaged MSE results of 30 runs obtained by the IDEA for the fusion of Kids image (Figure 4).

\begin{tabular}{|c|c|c|c|c|c|}
\hline Functions & CR/FR & 0.3 & 0.6 & 0.9 & 1.2 \\
\hline SF & 0.3 & 1.1284 & 1.1374 & 1.1447 & 1.1426 \\
& 0.6 & 1.1280 & 1.1307 & 1.1615 & 1.1348 \\
& 0.9 & 1.1283 & 1.1343 & 1.1346 & 1.1331 \\
& 1.2 & 1.1285 & 1.1536 & 1.1565 & 1.1420 \\
\hline VAR & 0.3 & 1.1990 & 1.2070 & 1.1992 & 1.2593 \\
& 0.6 & 1.1599 & 1.1788 & 1.1823 & 1.2404 \\
& 0.9 & 1.1725 & 1.1831 & 1.1686 & 1.1973 \\
& 1.2 & 1.1768 & 1.1766 & 1.1906 & 1.1723 \\
\hline SML & 0.3 & 1.5837 & 1.7084 & 1.7411 & 1.7438 \\
& 0.6 & 1.5629 & 1.6688 & 1.7413 & 1.7045 \\
& 0.9 & 1.5931 & 1.7435 & 1.7149 & 1.7604 \\
& 1.2 & 1.6693 & 1.7451 & 1.7853 & 1.7254 \\
\hline
\end{tabular}

(4) The comparison of the local search ability of the original DE algorithm and the IDEA applied in the fusion of Figure 6 (clock image) is shown in Table 3. This table presents the optimal block size and the number of searches in the initial intervals of the optimal block size in 10 experiments. The original DE algorithm only performs the search once, and the convergence is not robust. By contrast, the IDEA performs the search many times. The result clearly reveals that the local search ability of the IDEA is stronger than that of the original DE algorithm.

Referring to the results presented in Tables 2 and 3 as well as in Figures 7 to 9, all MES and PSER values, convergence result, as well as the global and local search ability of the IDEA are better than those of the original $\mathrm{DE}$ algorithm. We can therefore conclude that the IDEA performs better than the original $\mathrm{DE}$ algorithm in image fusion.

(5) The parameters of the IDEA are also analyzed. The fusion results of the Kids image using the IDEA are given in Table 4. The table shows the mean of the MES results of 30 replicates. The most suitable mutation factor that gives the best fitness value is 0.6 . The results also show that the 
Table 5 Absolute values of sharpness of the corresponding blocks when applying SF is applied $(64 \times 64)$.

\begin{tabular}{|c|c|c|c|}
\hline Numbers & Figure 4 & Figure 5 & Figure 6 \\
\hline 1 & 0.26 & 1.55 & 0.66 \\
2 & 0.26 & 1.55 & 0.66 \\
3 & 2.54 & 2.54 & 2.74 \\
4 & $\mathbf{5 . 4 3}$ & 2.72 & $\mathbf{6 . 0 3}$ \\
5 & $\mathbf{9 . 6 9}$ & 3.80 & $\mathbf{1 0 . 2 4}$ \\
6 & 10.23 & 4.22 & 10.60 \\
7 & 12.09 & 5.50 & 10.75 \\
8 & 12.21 & 5.97 & 11.20 \\
9 & 12.29 & 6.67 & 12.29 \\
10 & 13.19 & 7.72 & 12.80 \\
11 & 13.42 & 8.39 & 13.04 \\
12 & 13.42 & 9.86 & 13.19 \\
13 & 14.28 & 11.39 & 13.42 \\
14 & 15.69 & $\mathbf{1 1 . 9 8}$ & 15.72 \\
15 & 17.05 & $\mathbf{1 7 . 0 8}$ & 17.05 \\
16 & 19.62 & 19.91 & 19.62 \\
\hline
\end{tabular}

best crossover rate is obtained at 0.3 . The results also indicate that the best MES values are obtained when SF is used as a sharpness measure.

(3) Test 3: Comparison of the proposed method (multifocus image fusion using IDEA and ADBM)for multi-focus image fusion using the original DE algorithm and using ADBM.

(1) $\mathrm{TH}$ selection of the proposed method

The input images (Figures 4 to 6) $\mathrm{A}$ and $\mathrm{B}$ are divided into non-overlapping blocks with the size of and the absolute values of the sharpness of the corresponding blocks by applying the criterion function $\mathrm{SF}$ (Table 5). The values italicized and written in bold represent the greatest adjacent difference that can be used as the initial TH. As shown in the table, TH can set to 10 of Figure 4 (Kids), TH 17 for Figure 5 (Lena), TH 10 of Figure 6 (Clock).

(2) Comparison results and time cost of the three methods.

The quantitative comparison results of the two methods applied in Figures 4, 5 and 6 are presented in Table 6. The comparison employs the MSE and TIME metrics.

The calculated mean MES values (1.93, 0.43 and 0.09 for Figures 4, 5 and 6, respectively) of the IDEA and ADBM method are less than those of the original DE method (2.901, 1.60 and 1.16 for Figures 4, 5 and 6, respectively). Meanwhile, but the time cost (33.20, 35.58 and 25.68 for Figures 4,5 and 6, respectively) of the IDEA and ADBM method is much less than that of the original DE method (95.43, 51.86 and 44.46 for Figures 4, 5 and 6, respectively). The results indicate that the IDEA and ADBM method has better performance, requires shorter TIME, improves efficiency more effectively compared with the original DE algorithm. The experimental results also show that the calculated mean Time values of the IDEA and ADBM method are higher than those of the ADBM method for Figures 4 to 6.3. How-
Table 6 Comparison of the three methods.

\begin{tabular}{|l|l|c|c|c|c|c|}
\hline Images & Functions & Methods & F & CR & MSE & TIME \\
\hline $\begin{array}{l}\text { Figure } \\
4\end{array}$ & SF & Original DE & 0.3 & 0.3 & 2.90 & 95.43 \\
& & ADBM & - & - & 3.52 & 10.29 \\
& & IDEA and ADBM & 0.3 & 0.3 & 1.93 & 33.20 \\
\hline $\begin{array}{l}\text { Figure } \\
5\end{array}$ & SF & Original DE & 0.3 & 0.3 & 1.60 & 51.86 \\
& & ADBM & - & - & 5.55 & 30.37 \\
\hline $\begin{array}{l}\text { Figure } \\
6\end{array}$ & SF & Original DE & 0.3 & 0.3 & 1.16 & 44.46 \\
& & ADEA and ADBM & 0.3 & 0.3 & 0.43 & 35.58 \\
& & ADEA and ADBM & 0.3 & 0.3 & 0.09 & 25.68 \\
\hline
\end{tabular}

ever, the calculated mean MSE values of the IDEA and ADBM method are lower than those of the ADBM. As indicated by the results provided in Table 1 to 6 and Figures 1 to 6.8 , the IDEA and ADBM method can obtain better fusion effects, be calculated more efficiently, and consume less time compared with the original DE algorithm.

\section{Conclusion}

This thesis focuses on the fusion rules and algorithm performance of a multi-focus image fusion algorithm based on the original DE algorithm. To improve the fusion result, the IDEA is first utilized for the selection of the optimal block size. The extends block selection mechanism is also applied to determine the sharper image blocks with the same sharpness values. To improve the efficiency of image fusion, the IDEA is combined with the ADBM, which divides the boundary region only. A multi-focus images fusion using the IDEA and ADBM is thus established. First, the source images are divided once using the fixed size. The boundary region is then searched using the IDEA algorithm to find adaptive blocks. If the sharpness values of the corresponding blocks are equal, then the extends block mechanism is applied to determine the block with the highest sharpness value. Experimental results show that this algorithm can obtain better fusion effects, be calculated more efficiently, and consume less time.

\section{Acknowledgement}

This work is supported in part by the National Nature Science Foundation of China (No. 61103114), Fundamental Research Funds for the Central Universities (CDJZR13180089), and the Key Project of Chongqing Postgraduate Education Reform (No. yjg132027). 


\section{References}

[1] E.P. Baltsavias, Object extraction and revision by image analysis using existing geodata and knowledge: current status and steps towards operational systems, ISPRS Journal of Photogrammetry and Remote Sensing, 58, 129-151 (2004).

[2] Abramoff, M.D.; Magalhes, Paulo J.; Ram, Sunanda J., Image processing with ImageJ, Biophotonics international, 11, 36-42 (2004).

[3] Chun-Ming Tsai. Adaptive Local Power-Law Transformation for Color Image Enhancement. Applied Mathematics \& Information Sciences, 7, 2019-2026 (2013).

[4] V. Aslantas, R. Kurban, Fusion of multi-focus images using differential evolution algorithm.Expert Systems with Applications, 37, 8861-8870 (2010).

[5] Shutao Li, James T.Kwok, Yaonan Wang, Combination of images with diverse focuses using the spatial frequency, Information fusion, 2, 169-176 (2010).

[6] R.Stom,K.Priee, Differential evolution-a simple and efficient heuristic for global optimization over continuous spaces Journal of Global Optimization, 11, 341359 (1997).

[7] Jianyong Sun, Qingfu Zhang and Edward P.K, Tsang, DE/EDA: A new evolutionary algorithm for global optimization, Preprint submitted to Elsevier Science, 169, 249-262 (2005).

[8] Ji-Pyng Chiou, Chung-Fu Chang, Ant direction hybrid differential evolution for solving large capacitor placement problems, IEEE Transactions on power systems, 19, 1794-1800 (2004).

[9] Millie Pant, Musrrat Ali and V. P. Singh, Differential evolution using quadratic interpolation for initializing the population, Advance Computing Conference of the IEEE, 375-380 (2009).

[10] Zhang, X. M., Han, J. Q \& Liu, P. F, Restoration and fusion optimization scheme of multi-focus image using genetic search strategies, Optical Applicata, XXXV, 927-940 (2005).

[11] Qu Guagxiang,Xu Xiaoxiao,Multi-focus image fusion method based on pixel-level sub-block approach,Computer Engineering and Applications, 48, 155 (2012).

[12] Li Juan,Li Shutao,multi-focus images fusion based on adaptive block mechanism, Computer applications research Suppl, (2006).

[13] Ahmet M.Eskicioglu and Paul S.Fisher, Image quality measures and their performance, IEEE Transactions on communications, 43, 2959-2965 (1995).

[14] Jun Kong, Kaiyuan Zheng, Jingbo Zhang, Xue Feng,Multi-focus Image Fusion Using Spatial Frequency and Genetic Algorithm,IJCSNS International Journal of Computer Science and Network Security, 8, 220-224 (2008).

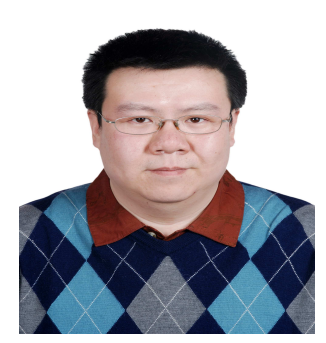

Feng Yong is professor at the College of Computer Science of Chongqing University, China. In 2000, he received his bachelor's degree in computer applied technology from Chongqing University. In 2003, he received his master's degree in computer systems organization from Chonqing University. In 2006, he received his $\mathrm{PhD}$ in computer software and theory from Chongqing University. From July 2007 to July 2010, he finished his postdoctoral research at the Control Science and Engineering Center of Chongqing University. In 2013, he was promoted to professor.

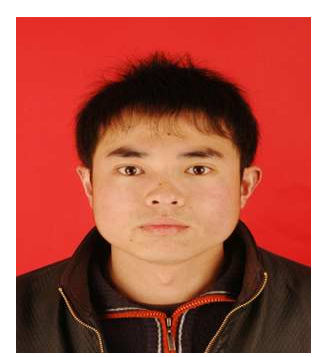

Li Tiezhu is a graduate student at Chongqing University and is mainly engaged in multi-focus image fusion.

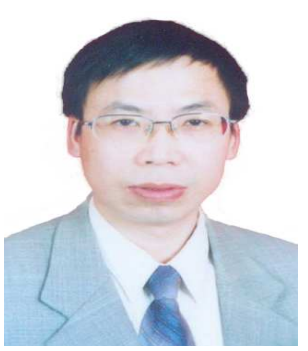

Zhou Shangbo received his BSc degree in math from Gangxi National College in 1985, MSc degree in math from Sichuan University in 1991, and his $\mathrm{PhD}$ degree in circuit and system from Electronic Science and Technology University. From 1991 to 2000, he worked at the Chongqing Aerospace

Electronic and Mechanical Technology Design Research Institute. Since 2003, he has been with the Department of Computer Science and Engineering, where he is now an professor. His current research interests include artificial neural networks, physical engineering simulation, visual object tracking, and nonlinear dynamical system. 